\title{
MAXIMIZACIÓN DE LA EFICIENCIA A CARGAS PARCIALES DE UNA CENTRAL HIDROELECTRICA MULTIGRUPO INTEGRADA AL SISTEMA INTERCONECTADO NACIONAL
}

\author{
MAXIMIZING ENERGY EFFICIENCY IN PARTIAL LOADS OF A \\ MULTIGROUP HYDROELECTRIC POWER STATION INTEGRATED \\ WITHIN THE NATIONAL INTERCONNECTED SYSTEM
}

\author{
Salome Gonzales Chávez ${ }^{11}$, Miguel Rosas Maximiliano ${ }^{22}$, Alex Poccomo Coquil ${ }^{33}$
}

\begin{abstract}
RESUMEN
En una Central Hidroeléctrica multigrupo que opera a cargas parciales, la máxima eficiencia de cada grupo y el de la combinación aleatorias de estos, difieren de la eficiencia de diseño de la central a plena carga. Estas centrales están condicionadas a operar a cargas parciales, tanto en épocas de estiaje como en tramos de sobreoferta eléctrica del sistema interconectado nacional. El periodo de estiaje en Perú alcanza a nueve meses del año, situación que implica pérdidas económicas debido a la inadecuada regulación del caudal y elección de grupo, a condiciones de eficiencias por debajo de sus valores óptimos.

El objetivo de este trabajo es el desarrollo de un proceso de optimización de la eficiencia de una Central Hidroeléctrica multigrupo, para cualquier nivel de potencia a generar en el programa diario de generación interconectada.

Como resultados se obtiene la maximización de la eficiencia y combinación de turbinas-grupo para cada potencia de salida de la central fuera de sus condiciones nominales; asimismo se construye un programa computacional en Matlab que alimenta al sistema automatizado de la central. Su aplicación está permitiendo reducir costos de generación y disponibilidad de la central, a su vez conforma un patrón metodológico de uso en otras centrales hidroeléctricas multigrupo.
\end{abstract}

Palabras clave: Central hidroeléctrica multigrupo, Cargas parciales, Maximización, Despacho económico, Estiaje, Sistema interconectado nacional.

\begin{abstract}
In multigroup hydroelectric plant operating at partial loads, the maximum each group efficiency and the random combination of these, differ from the design efficiency of the plant at full load. These plants are conditioned to operate at partial loads, both in times of drought and periods of oversupply national electric grid. The period of drought in Peru is nine months per year, a situation that involves economic losses due to inadequate flow regulation and group choice efficiencies conditions below their optimum values. The aim of this work is the development optimizing efficiency process of a multigroup hydroelectric power plant, for any level of power generated in the daily interconnected generation program. The results are maximizing turbine efficiency and combined-group for each power output of the plant, outside its nominal conditions, also a computer program is constructed in Matlab to integrate the automated system of hydroelectric power plant.

Its application can reduce generation costs and increases plant availability; also forms a methodological pattern of use in other multigroup hydroelectric plants.
\end{abstract}

Key words: Multigroup hydroelectric plant, Partial loads, Maximization, Economic dispatch, Time of drought, National grid.

\footnotetext{
La Revista Científica TECNIA protege los derechos de autor bajo la Licencia 4.0 de Creative Commons: Attribution 4.0 International (CC BY 4.0)

${ }^{1}$ Doctor docente investigador de la Facultad de Ingeniería Mecánica-Universidad Nacional de Ingeniería

${ }^{2}$ Ing. egresado de la Facultad de Ingeniería Mecánica-Universidad Nacional de Ingeniería,

${ }^{3}$ Ing Egresado de la Facultad de Ingeniería Mecánica-Universidad Nacional de Ingeniería
} 


\section{PLANTEAMIENTO DEL PROBLEMA Y OBJETIVOS}

\section{Planteamiento de problema}

Una central hidroeléctrica de varios grupos, en su operación diaria presenta problemas de generación eficiente tanto en épocas de estiaje, como durante los recortes de potencia por sobreoferta eléctrica del sistema interconectado; comúnmente se procede a regular el caudal de cada grupo bajo forma aleatoria, muchas veces fuera de las mejores condiciones de rendimiento de cada unidad, combinación de unidades y del conjunto. Ello se agrava en la medida que aumentan las pérdidas transversales con el avance de la vida útil de la central [1].

La importancia de este trabajo se enmarca en el desarrollo de una metodología y herramienta para la optimización operativa de una central hidroeléctrica multigrupo, maximizando el rendimiento del conjunto que opera a cargas parciales dentro del despacho diario al sistema interconectado. Bajo estas condiciones se logran reducir costos de generación en la operación de corto plazo [2], consecuentemente mejoras en los precios spot dentro de un gran sistema de generación interconectado [3].

\section{OBJETIVOS}

Desarrollo de una metodología y aplicación informatizada de optimización de la potencia eléctrica a generar de cada unidad turbogeneradora de una Central Hidroeléctrica multigrupo, tal que maximice la eficiencia de planta de la central cuando ésta despacha potencias menores que su potencia nominal o de diseño, dentro de un programa diario de generación interconectada. Se toma como aplicación típica representativa a la C.H. Callahuanca-Perú de $80 \mathrm{MW}$ de potencia instalada. Como producto se tiene:

- La elaboración de un procedimiento para la determinación de la condición óptima de distribución de potencia entre las unidades turbogeneradoras, para cargas parciales de generación de la central.

- El desarrollo de un programa en Matlab, amplio y versátil en la disposición y número de grupos de una central, que ha de ser integrado al sistema automático de control de la central.

\section{METODOLOGIA}

El procedimiento inicia con las pruebas de potencias y rendimientos a diversas condiciones de carga practicadas a la central en estudio. En este caso las pruebas se han realizado mediante el Método Termodinámico para cada uno de los cuatro grupos de la C. H. Callahuanca. El Método Termodinámico, en el cual adicionalmente a las pruebas realizadas con el método convencional, se mide la diferencia de temperaturas del agua entre la entrada y salida de cada turbina hidráulica, con la finalidad de estimar adicionalmente el efecto energético del pequeño cambio de temperatura entre su entrada y salida. Se toma como referencia las recomendaciones de la Norma IEC 60041 y ASME PTC 18, [4].

A partir de estas mediciones se elaboran las expresiones matemáticas de correspondencia entre las variables objetivo de optimización: potencias, caudales y alturas. Los ajustes de curvas de estas relaciones servirán como elementos de referencia que identifican la situación energética real actual de la central hidroeléctrica.

El sistema óptimo de distribución de potencia se logra seleccionando a máximo rendimiento los caudales de entrega de cada unidad turbogeneradora para los diversos valores de potencia de despacho de la central, desde el valor mínimo cubierto por un solo grupo, pasando por combinaciones de grupos, hasta cuando la central opera con el total de grupos. Los caudales se optimizan tomando en cuenta la semejanza dinámica de cada turbina a altura de diseño, esto es a igualdad de cifra de presión y cifra de caudal. Existen otras metodologías alternativas para casos específicos $[5,6,7]$.

\section{PRUEBAS DE REFERENCIA}

De las pruebas realizadas para cada grupo, se obtienen los siguientes resultados, que una vez ajustados sirven como valores patrón a posteriori para el cálculo de la condición óptima de distribución de potencia de las unidades turbogeneradoras, para cualquier condición instantánea de generación del conjunto central. En efecto, en las tablas 1 y 2 se muestran los resultados de las pruebas de los grupos 1 y 2: potencias, caudales, saltos hidráulicos, eficiencias y otros, obtenidos variando el caudal 
mediante la carrera de las agujas de los inyectores de cada turbina.

Tabla 1. Resultado de pruebas por el Método Termodinámico del grupo G1.

\begin{tabular}{|c|c|c|c|c|c|c|c|c|c|}
\hline \multicolumn{10}{|c|}{$\begin{array}{l}\text { Central Hidroeléctrica de Callahuanca - Grupo Nro. } 1 \\
\text { Pruebas de eficiencia con método termodinámico - Salto neto de referencia }=415 \mathrm{~m}\end{array}$} \\
\hline Prueba & $\begin{array}{c}\text { Potencia } \\
\text { eléctrica } \\
\text { activa }\end{array}$ & $\begin{array}{c}\text { Pérdidas } \\
\text { del } \\
\text { generador }\end{array}$ & $\begin{array}{c}\text { Eficiencia } \\
\text { del } \\
\text { generador }\end{array}$ & \begin{tabular}{|c|} 
Caudal \\
volumétrico \\
del grupo
\end{tabular} & $\begin{array}{c}\text { Salto } \\
\text { hidráulico } \\
\text { neto }\end{array}$ & $\begin{array}{l}\text { Potencia } \\
\text { hidráulica }\end{array}$ & $\begin{array}{l}\text { Potencia } \\
\text { mecánica }\end{array}$ & $\begin{array}{c}\text { Eficiencia } \\
\text { hidráulica } \\
\text { rodete }\end{array}$ & $\begin{array}{c}\text { Eficiencia } \\
\text { de la } \\
\text { turbina }\end{array}$ \\
\hline & {$[\mathrm{kW}]$} & {$[\mathrm{kW}]$} & {$[\%]$} & {$[\mathrm{m} 3 / \mathrm{s}]$} & {$[\mathrm{m}]$} & {$[\mathrm{kW}]$} & {$[\mathrm{kW}]$} & [\%] & {$[\%]$} \\
\hline$\overline{N^{\circ} 1}$ & 15664.00 & 254.09 & 98.40 & 4.563 & 417.36 & 18644.43 & 15925.09 & 85.415 & 85.377 \\
\hline$\overline{\mathrm{N}^{\circ} 2}$ & 14930.75 & 245.23 & 98.38 & 4.286 & 417.95 & 17583.47 & 15182.98 & 86.348 & 86.308 \\
\hline $\mathrm{N}^{\circ} 3$ & 1343 & 234.46 & 98.28 & 3.789 & 421.38 & 1567 & 13672.13 & 87.226 & 87.181 \\
\hline $\mathrm{N}^{\circ} 4$ & 11952.59 & 223.07 & 98.17 & 3.334 & 424.98 & 13910.24 & 121 & .581 & 87.530 \\
\hline $\mathrm{N}^{\circ} 5$ & 8952.57 & 202.72 & 97.79 & 2.488 & 429.52 & 10490.65 & 9162.28 & 87.338 & 87.271 \\
\hline $\mathrm{N}^{\circ} 6$ & 1343 & 236.44 & 98.27 & 3.773 & 422.41 & 15643.88 & 13676.13 & 7.422 & 87.377 \\
\hline Prueba & $\begin{array}{l}\text { Error } \\
\text { total }\end{array}$ & $\begin{array}{l}\text { Eficiencia } \\
\text { del grupo }\end{array}$ & $\begin{array}{l}\text { Caudal al } \\
\text { salto Href }\end{array}$ & $\begin{array}{c}\text { Potencia } \\
\text { mecánica a } \\
\text { Href }\end{array}$ & $\begin{array}{c}\text { Potencia } \\
\text { eléctrica } \\
\text { a Href }\end{array}$ & $\begin{array}{c}\text { Pérdidas } \\
\text { en la } \\
\text { tubería }\end{array}$ & $\begin{array}{c}\text { Carrera } \\
\text { media de } \\
\text { agujas }\end{array}$ & $\begin{array}{c}\text { Carrera } \\
\text { relativa } \\
\text { agujas }\end{array}$ & $\begin{array}{c}\text { Coeficiente } \\
\text { de caudal } \\
\text { agujas }\end{array}$ \\
\hline & [\%] & [\%] & {$[\mathrm{m} 3 / \mathrm{s}]$} & {$[\mathrm{kW}]$} & {$[\mathrm{kW}]$} & {$[\mathrm{m}]$} & {$[\mathrm{mm}]$} & & \\
\hline $\mathrm{N}^{\circ} 1$ & $0.907 \%$ & 84.014 & 4.550 & 15783.46 & 15531.52 & 18.491 & 128.160 & 0.567 & 0.628 \\
\hline$\overline{N^{\circ} 2}$ & $0.833 \%$ & 84.914 & 4.271 & 15015.76 & 14773.12 & 17.741 & 108.036 & 0.478 & 0.589 \\
\hline $\mathrm{N}^{\circ} 3$ & $0.940 \%$ & 85.685 & 3.761 & 13355.85 & 13126.70 & 14.056 & 86.616 & 0.383 & 0.519 \\
\hline $\mathrm{N}^{\circ} 4$ & $0.691 \%$ & 85.927 & 3.295 & 11749.20 & 11533.95 & 10.896 & 70.524 & 0.312 & 0.454 \\
\hline $\mathrm{N}^{\circ} 5$ & $0.820 \%$ & 85.338 & 2.446 & 8694.99 & 8502.47 & 6.968 & 54.008 & 0.239 & 0.337 \\
\hline$\overline{N^{\circ} 6}$ & $0.572 \%$ & 85.865 & 3.740 & 13311.04 & 13080.79 & 14.038 & 86.350 & 0.382 & 0.516 \\
\hline
\end{tabular}

Tabla 2. Resultado de pruebas por el Método Termodinámico del grupo G2.

\begin{tabular}{|c|c|c|c|c|c|c|c|c|c|}
\hline \multicolumn{10}{|c|}{$\begin{array}{c}\text { Central Hidroeléctrica de Callahuanca - Grupo Nro. } 2 \\
\text { Pruebas de eficiencia con método termodinámico - Salto neto de referencia }=415 \mathrm{~m}\end{array}$} \\
\hline uet & $\begin{array}{l}\text { Potencia } \\
\text { a eléctrica }\end{array}$ & $\begin{array}{c}\text { Perdidas } \\
\text { del } \\
\text { generador }\end{array}$ & \begin{tabular}{|c|} 
Eficiencia \\
del \\
generador
\end{tabular} & $\begin{array}{c}\text { Caudal } \\
\text { volumétrico }\end{array}$ & \begin{tabular}{|c|} 
Salto \\
ohidráulico
\end{tabular} & $\begin{array}{c}\text { Potencia } \\
\text { hidráulica }\end{array}$ & \begin{tabular}{|l|} 
Potencia \\
mecánica
\end{tabular} & Eficiencia & \begin{tabular}{|c|c} 
Eficiencia \\
de la \\
turbina
\end{tabular} \\
\hline & {$[\mathrm{kW}]$} & {$[\mathrm{kW}]$} & [\%] & {$[\mathrm{m} 3 / \mathrm{s}]$} & [m] & {$[\mathrm{kW}]$} & {$[\mathrm{kW}]$} & [\%] & [\%] \\
\hline${ }^{\circ} 1$ & 15660.08 & 260.55 & 98.36 & 4.582 & 415.51 & 18642.12 & 15927.63 & 85.439 & 85.401 \\
\hline${ }^{\circ} 2$ & 14948 & 254.02 & 98.33 & 4.30 & 417.85 & & 152 & 86.2 & 1802 \\
\hline $\mathrm{N}^{\circ} 3$ & 1349 & 244.86 & 98.22 & 3.822 & 422.03 & 15 & 13 & 87.075 & 0 \\
\hline$N^{\circ} 4$ & & 266 & 98.39 & 4.8 & & & & & \\
\hline${ }^{\circ} 5$ & 92 & 228 & 97.60 & 2.6 & 429 & & & & \\
\hline $\mathrm{N}^{\circ} 6$ & 13296.01 & 245.70 & 98.19 & 3.770 & 422.32 & 155 & 13 & 86. & 86.86 \\
\hline ueba & $\begin{array}{l}\text { Error } \\
\text { total }\end{array}$ & $\begin{array}{l}\text { Eficie } \\
\text { del g }\end{array}$ & $\mid \begin{array}{c}\text { Caudal al } \\
\text { salto Href }\end{array}$ & $\begin{array}{c}\text { Potencia } \\
\text { mecánica a } \\
\text { Href }\end{array}$ & $\begin{array}{c}\text { Potencia } \\
\text { eléctrica } \\
\text { a Href }\end{array}$ & $\begin{array}{c}\text { Pérdidas } \\
\text { en la } \\
\text { tubería }\end{array}$ & \begin{tabular}{|c|} 
Carrera \\
media de \\
agujas
\end{tabular} & $\begin{array}{c}\text { Carrera } \\
\text { relativa } \\
\text { agujas }\end{array}$ & $\begin{array}{c}\text { Coeficiente } \\
\text { de caudal }\end{array}$ \\
\hline & [\%] & [\%] & {$[\mathrm{m} 3 / \mathrm{s}]$} & {$[\mathrm{kW}]$} & {$[\mathrm{kW}]$} & [m] & {$[\mathrm{mm}]$} & & \\
\hline 1 & $0.667 \%$ & 84.004 & 4.579 & & 563 & & & & \\
\hline & & 96 & 4.2 & & & & & & \\
\hline 3 & $22 \%$ & 80 & 3.790 & 13 & 9 & & & & 23 \\
\hline $\mathrm{N}^{\circ} 4$ & $23 \%$ & 83.403 & 4.824 & 16614.23 & 16346.66 & 22.294 & 143.424 & 0.634 & 0.666 \\
\hline $\mathrm{N}^{\circ} 5$ & $0.541 \%$ & 84.726 & 2.558 & 9025.10 & 8808.24 & 6.561 & 48.528 & 0.215 & 0.353 \\
\hline $\mathrm{N}^{\circ} 6$ & $0.648 \%$ & 85.290 & 3.737 & 13191.22 & 12951.88 & 13.989 & 86.400 & 0.382 & 0.516 \\
\hline
\end{tabular}




\section{AJUSTE DE CURVAS DE RENDIMIENTO DE CADA GRUPO}

La obtención de la ecuación de ajuste de las curvas características para cada grupo, se realiza tomando como base la maximización del coeficiente estadístico de autodeterminación $\mathrm{R}^{2}$. Así, en las figuras 1 a la 4 se ilustran las curvas de rendimiento vs potencia eléctrica generada, de los cuatro grupos de la C. H Callahuanca.

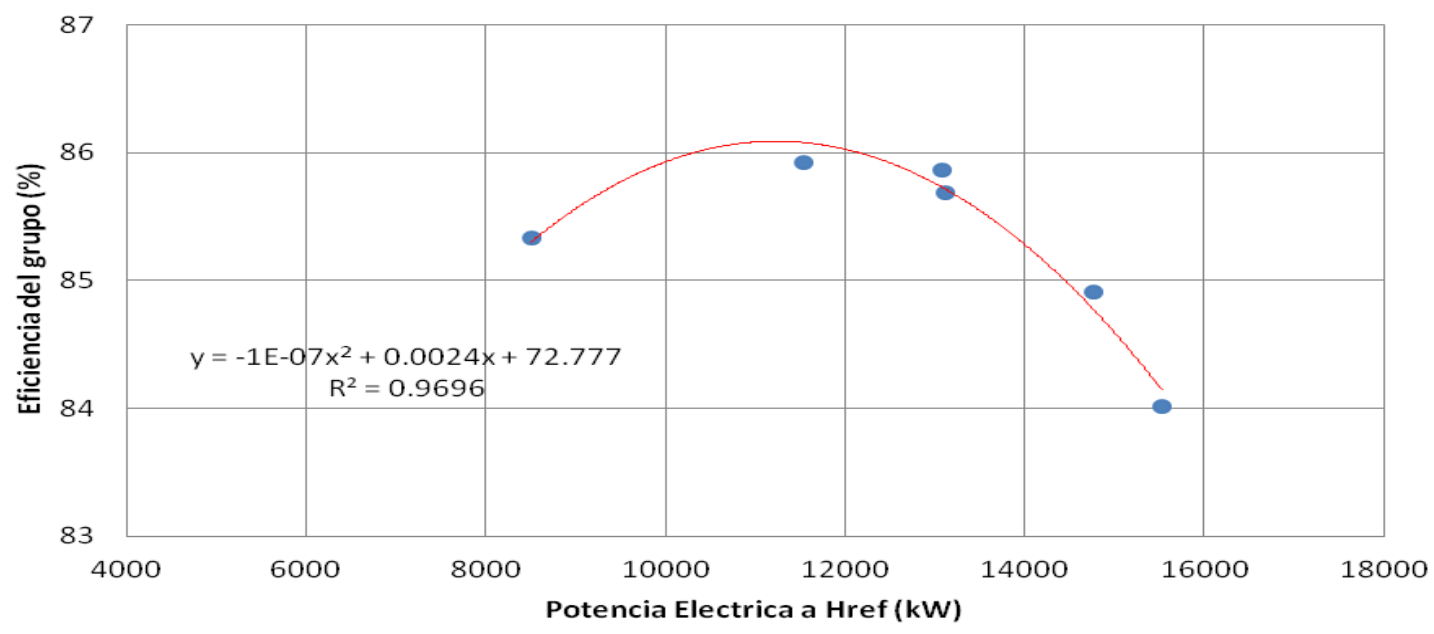

Fig. 1 Curva de Rendimiento vs Potencia de salida del Grupo 1.

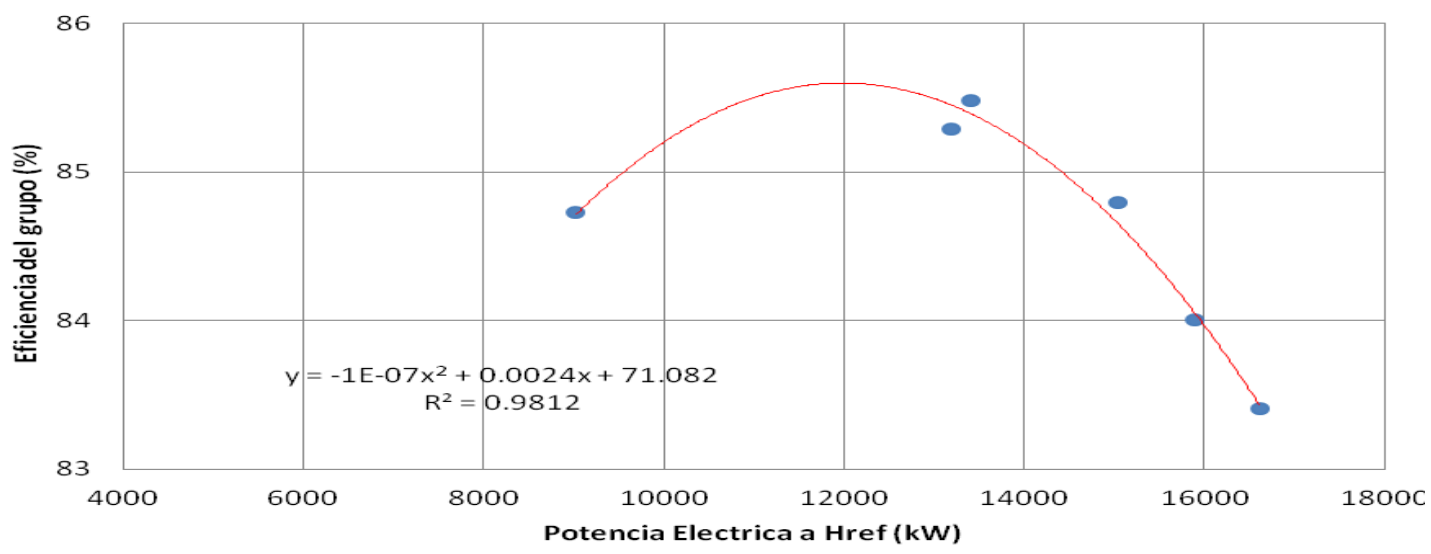

Fig. 2 Curva de Rendimiento vs Potencia de salida del Grupo 2.

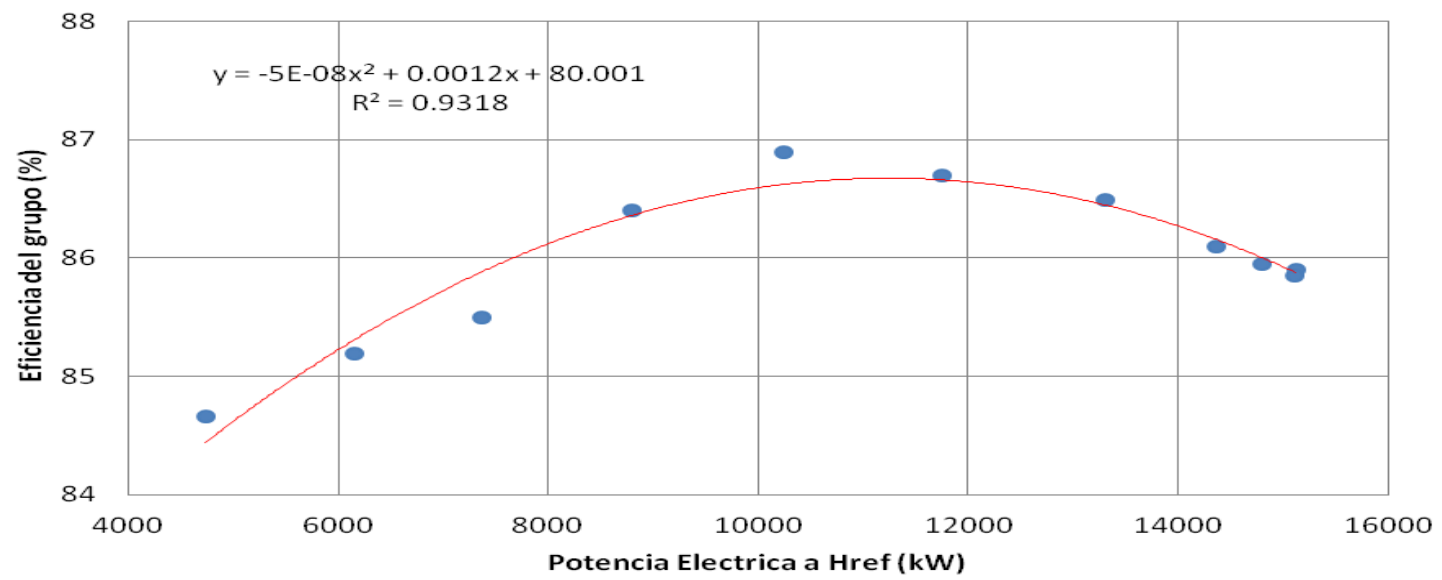

Fig. 3 Curva de Rendimiento vs Potencia de salida del Grupo 3. 


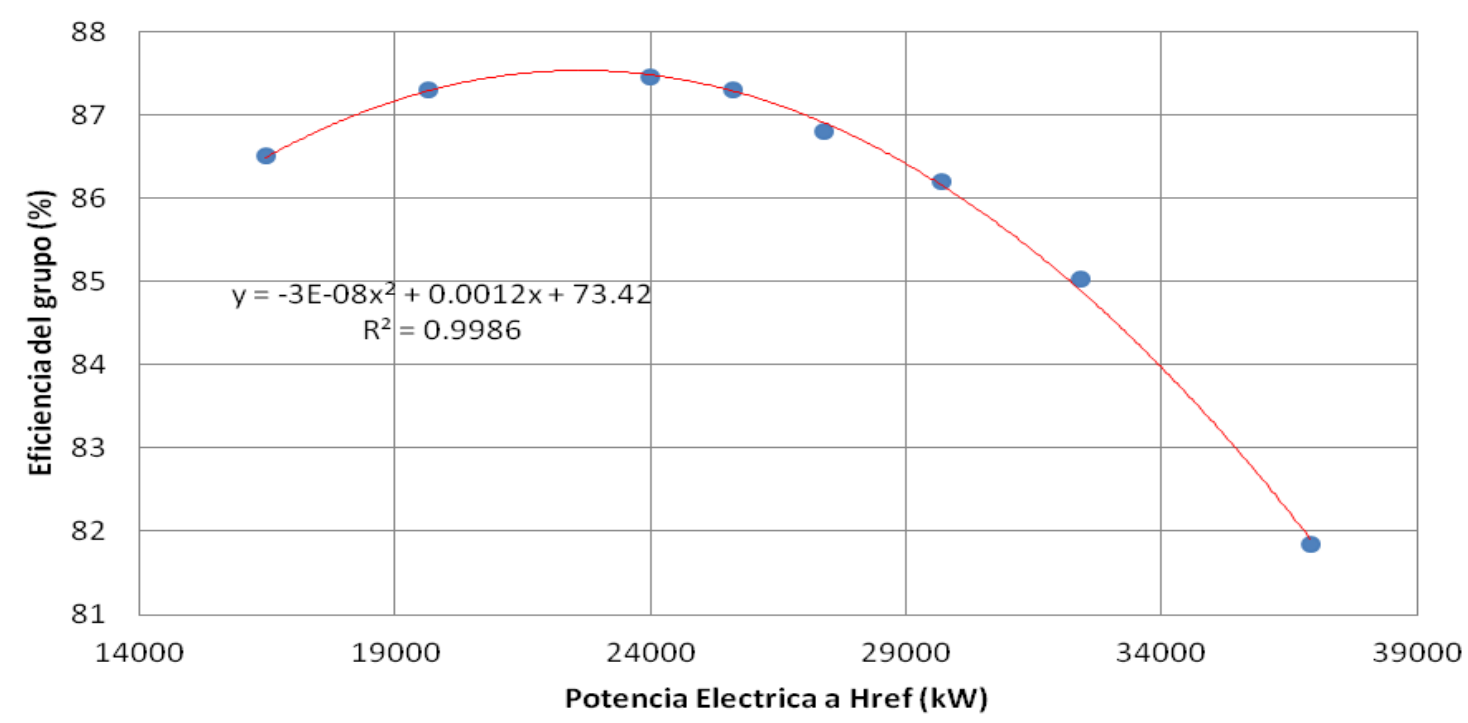

Fig. 4 Curva de Rendimiento vs Potencia de salida del Grupo 4.

\begin{tabular}{|c|c|c|}
\hline \multirow{4}{*}{$\begin{array}{l}\text { Potencia de turbina } \\
\text { vs } \\
\text { Potencia generada }\end{array}$} & Pt1 & $=+0.0001573353 . \operatorname{Pg} 1^{2}+1.0036492474 . \operatorname{Pg} 1+0.1644175442$ \\
\hline & $\mathrm{Pt} 2$ & $=+0.0004625344 \cdot \operatorname{Pg} 2^{2}+0.9935158785 \cdot \operatorname{Pg} 2+0.2557514780$ \\
\hline & Pt3 & $=+0.0004625344 \cdot \operatorname{Pg} 3^{2}+0.9935158785 \cdot \operatorname{Pg} 3+0.2557514780$ \\
\hline & $\mathrm{Pt} 4$ & $=+0.0002485447 \cdot \operatorname{Pg} 4^{2}+0.9966740929 \cdot \operatorname{Pg} 4+0.3921649701$ \\
\hline \multirow{4}{*}{$\begin{array}{l}\text { Potencia generada } \\
\text { vs. Potencia de } \\
\text { turbina }\end{array}$} & $\operatorname{Pg} 1$ & $=-0.0001540267 . \mathrm{Pt} 1^{2}+0.9963975319 . \mathrm{Pt} 1-0.1637610837$ \\
\hline & $\operatorname{Pg} 2$ & $=-0.0004550438 . \mathrm{Pt} 2^{2}+1.0065517232 . \mathrm{Pt} 2-0.2565524438$ \\
\hline & $\mathrm{Pg} 3$ & $=-0.0004550438 . \mathrm{Pt} 3^{2}+1.0065517232 . \mathrm{Pt} 3-0.2565524438$ \\
\hline & $\mathrm{Pg} 4$ & $=-0.0002416864 . \mathrm{Pt} 4^{2}+1.0032962124 . \mathrm{Pt} 4-0.3915823146$ \\
\hline \multirow{4}{*}{$\begin{array}{c}\text { Altura neta vs. } \\
\text { Potencia de turbina }\end{array}$} & Hn1 & $=-0.0739423033 . P t 1^{2}-0.0222323839 . P t 1+435.9995219709$ \\
\hline & $\mathrm{Hn} 2$ & $=-0.0994822462 . \mathrm{Pt} 2^{2}+0.3178416950 . \mathrm{Pt} 2+435.9350051294$ \\
\hline & Hn3 & $=-0.0994822462 . \mathrm{Pt} 3^{2}+0.3178416950 . \mathrm{Pt} 3+435.9350051294$ \\
\hline & $\mathrm{Hn} 4$ & $=-0.0419425840 . \mathrm{Pt} 4^{2}+0.4572160795 . \mathrm{Pt} 4+437.6772866504$ \\
\hline \multirow{4}{*}{$\begin{array}{c}\text { Altura neta } \\
\text { vs. } \\
\text { Caudal }\end{array}$} & Hn1 & $=-0.7335166984 . Q 1^{2}-0.9160836055 . Q 1+436.0549640843$ \\
\hline & $\mathrm{Hn} 2$ & $=-0.9792097475 . \mathrm{Q} 2^{2}+0.0487422372 . \mathrm{Q} 2+435.9985788147$ \\
\hline & $\mathrm{Hn} 3$ & $=-0.9792097475 . \mathrm{Q}^{2}+0.0487422372 . \mathrm{Q} 3+435.9985788147$ \\
\hline & $\mathrm{Hn} 4$ & $=-0.3434438425 . \mathrm{Q} 4^{2}+0.1545614354 . \mathrm{Q} 4+438.0711502046$ \\
\hline \multirow{4}{*}{$\begin{array}{l}\text { Caudal } \\
\text { vs } \\
\text { Potencia }\end{array}$} & Q1 & $=+0.0054132677 . \mathrm{Pt}^{2}+0.1621373674 . \mathrm{Pt} 1+0.6300996175$ \\
\hline & Q2 & $=+0.0050572833 . \mathrm{Pt} 2^{2}+0.1681334147 . \mathrm{Pt} 2+0.6298762384$ \\
\hline & Q3 & $=+0.0050572833 . \mathrm{Pt} 3^{2}+0.1681334147 . \mathrm{Pt} 3+0.6298762384$ \\
\hline & Q4 & $=+0.0031718483 . \mathrm{Pt} 4^{2}+0.1296333667 . \mathrm{Pt} 4+1.5296851572$ \\
\hline \multirow{4}{*}{$\begin{array}{l}\text { Potencia } \\
\text { vs } \\
\text { Caudal }\end{array}$} & $\mathrm{P} 1$ & $=-0.2102694188 . \mathrm{Q} 1^{2}+4.8595663752 . \mathrm{Q} 1-1.9436257178$ \\
\hline & $\mathrm{P} 2$ & $=-0.1889064520 . \mathrm{Q} 2^{2}+4.7492662947 . \mathrm{Q} 2-1.8911592924$ \\
\hline & $\mathrm{P} 3$ & $=-0.1889064520 . \mathrm{Q}^{2}+4.7492662947 . \mathrm{Q} 3-1.8911592924$ \\
\hline & $\mathrm{P} 4$ & $=-0.1143053721 . \mathrm{Q} 4^{2}+5.0628324417 . \mathrm{Q} 4-4.0210755320$ \\
\hline
\end{tabular}




\section{CÁLCULO Y ANALISIS DE RESULTADOS}

\section{Determinación de las Expresiones Matemáticas de Ajuste}

Se elaboran las ecuaciones de correspondencia matemática entre las variables objetivo, deducidas de los resultados de mediciones por el método termodinámico. De las correlaciones realizadas se

\section{SISTEMA ÓPTIMO DE DISTRIBUCIÓN DE POTENCIA DE LA CENTRAL}

El sistema óptimo de distribución de potencia se obtiene seleccionando los caudales de entrega de cada unidad turbogeneradora al mayor rendimiento correspondiente a la carga total solicitada a la central, en cualquier momento y con la disposición de un grupo, la combinación de éstos o el conjunto total de la central.

El rango de potencia de despacho va desde el valor mínimo cubierto por un solo grupo hasta cuando la central opera con el total de grupos. Así, el cálculo propuesto se realiza bajo el algoritmo secuencial siguiente:

a) Se tiene la combinación de potencias en el generador (Pg1, Pg2, Pg3,..,Pgi).

b) Se calcula sus respectivas potencias en turbina (Pt1, Pt2, Pt3..., Pti) mediante las relaciones $\mathrm{Pt}=\mathrm{F}(\mathrm{Pg})$ de cada grupo.

c) Se calcula sus respectivas alturas netas (Hn1, $\mathrm{Hn} 2$, Hn3 ... Hni) en función de sus respectivas potencias de turbina (Pt1, Pt2, Pt3..., Pti), mediante la relación $\mathrm{Hn}=\mathrm{G}(\mathrm{Pt})$.

d) Con las alturas netas Hni y con la altura de referencia Hsp (436 para G1, G2 y G3 o 438 para G4), se recalculan las potencias de determinan expresiones matemáticas de la forma cuadrática: $a \cdot x^{2}+b \cdot x+c$.

Cuando se realice una nueva prueba y mediciones en la central para la actualización de su performance, solamente se cambiarán los respectivos coeficientes de cada ecuación. Como se muestran en la Tabla precedente, las ecuaciones generadas para las cuatro unidades de la Central Hidroeléctrica de Callahuanca.

turbina en cada grupo Psp1, Psp2,..Pspi, mediante la relación de semejanza: $\mathrm{Psp}=\mathrm{Pt}$ $(\mathrm{Hsp} / \mathrm{Hn})^{1.5}$

e) Con estas potencias Psp se calculan los caudales respectivos mediante las relaciones $\mathrm{Qsp}=\mathrm{J}(\mathrm{Psp})$ de cada grupo

f) Con las alturas netas Hn y Hsp (436 para G1, G2 y G3 o 438 para G4) se recalculan los caudales Q'1, Q'2 y Q'3 mediante la relación de semejanza: $\mathrm{Q}^{\prime}=\mathrm{Qsp}(\mathrm{Hn} / \mathrm{Hsp}) 0.5$

g) Se recalcula la altura neta de cada grupo (Hn), en función de Q'i mediante la relación H'n = H(Q'T).

h) Con esta nueva altura neta se repite desde el paso (d) reiteradamente hasta lograr que la diferencia entre las Alturas netas entre una iteración consecutiva H'n sea menor a $0.05 \mathrm{~m}$. Se verifica que es suficiente no muchas iteraciones para lograr la convergencia de los valores de alturas netas.

i) La combinación de caudales Q'1, Q'2,..., Q’i que logra la condición anterior son los caudales óptimos correspondientes a la combinación de potencias (a).

En la figura 5 se presenta el diagrama de flujo que sintetiza el proceso de cálculo para la obtención de las condiciones óptimas de operación de la central en estudio. 


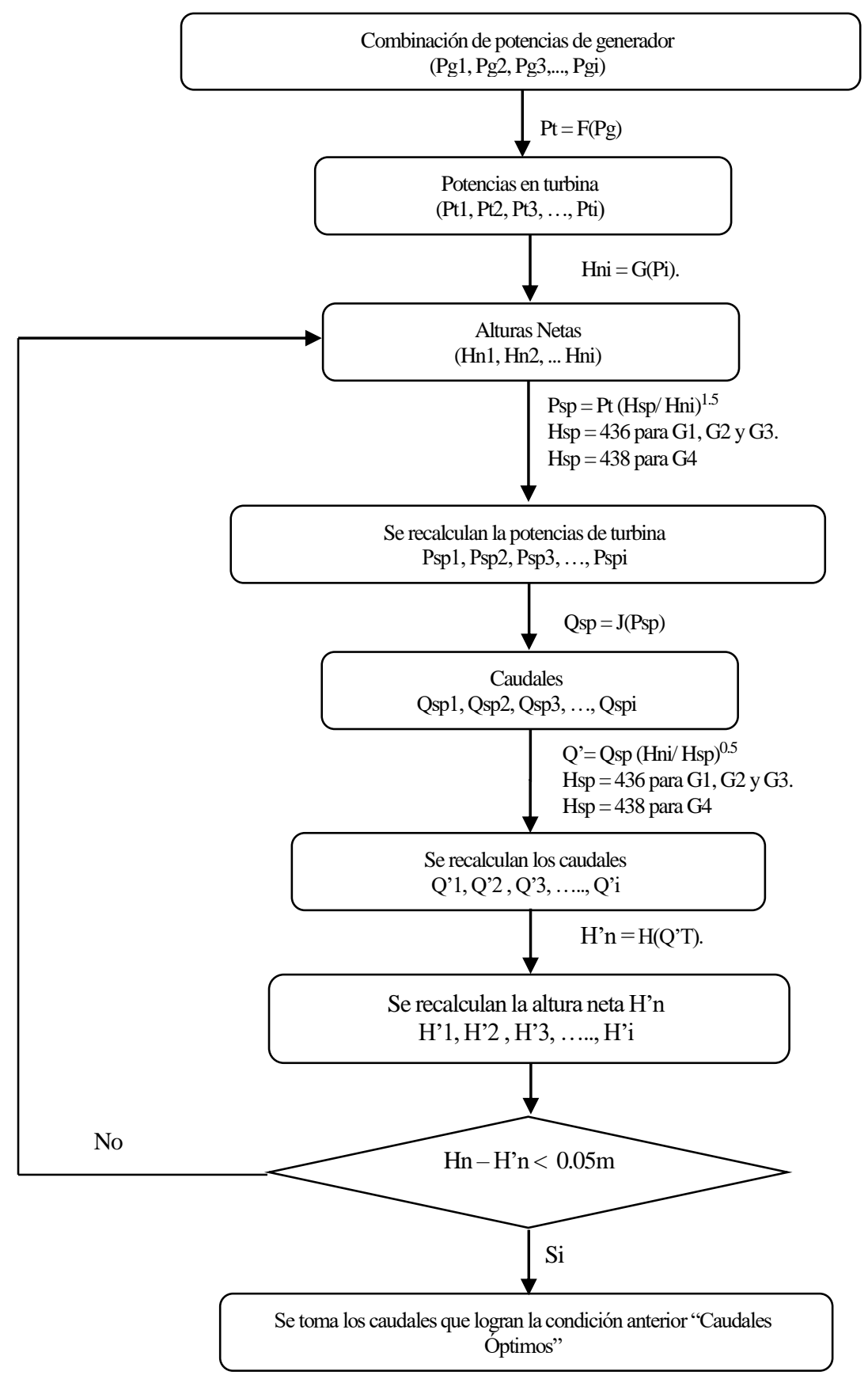

Fig. 5 Diagrama de Flujo del proceso.

\section{DESARROLLO DE PROGRAMA DE CÁLCULO DE OPTIMIZACIÓN EN MATLAB}

Uno de los objetivos del presente trabajo es el diseño de un programa computacional que sea versátil y se pueda extender su uso a cualquier central hidroeléctrica que posea más de una unidad o grupo de generación, a fin de alcanzar una operación diaria eficiente y económica que garantice el buen uso del recurso hídrico en periodos de estiaje así como la competitividad cuando hay sobreoferta hidroeléctrica.

Entre las principales características se tienen:

- Modificación de los coeficientes de las ecuaciones características de potencias, caudales y alturas 
- Selecciona las unidades que se encuentran disponibles con la finalidad de aperar de manera óptima sólo con aquellos grupos que se encuentran operativos al momento.

- Tamaño elegible de convergencia y partición contable de potencia generada de la central, para el cálculo iterativo de los caudales de cada grupo correspondientes al mejor arreglo de potencias a mayor rendimiento.

- Salidas gráficas y tabuladas en entorno Matlab y con exportación a MS Excel (ver figuras 6,7 y 8 ).

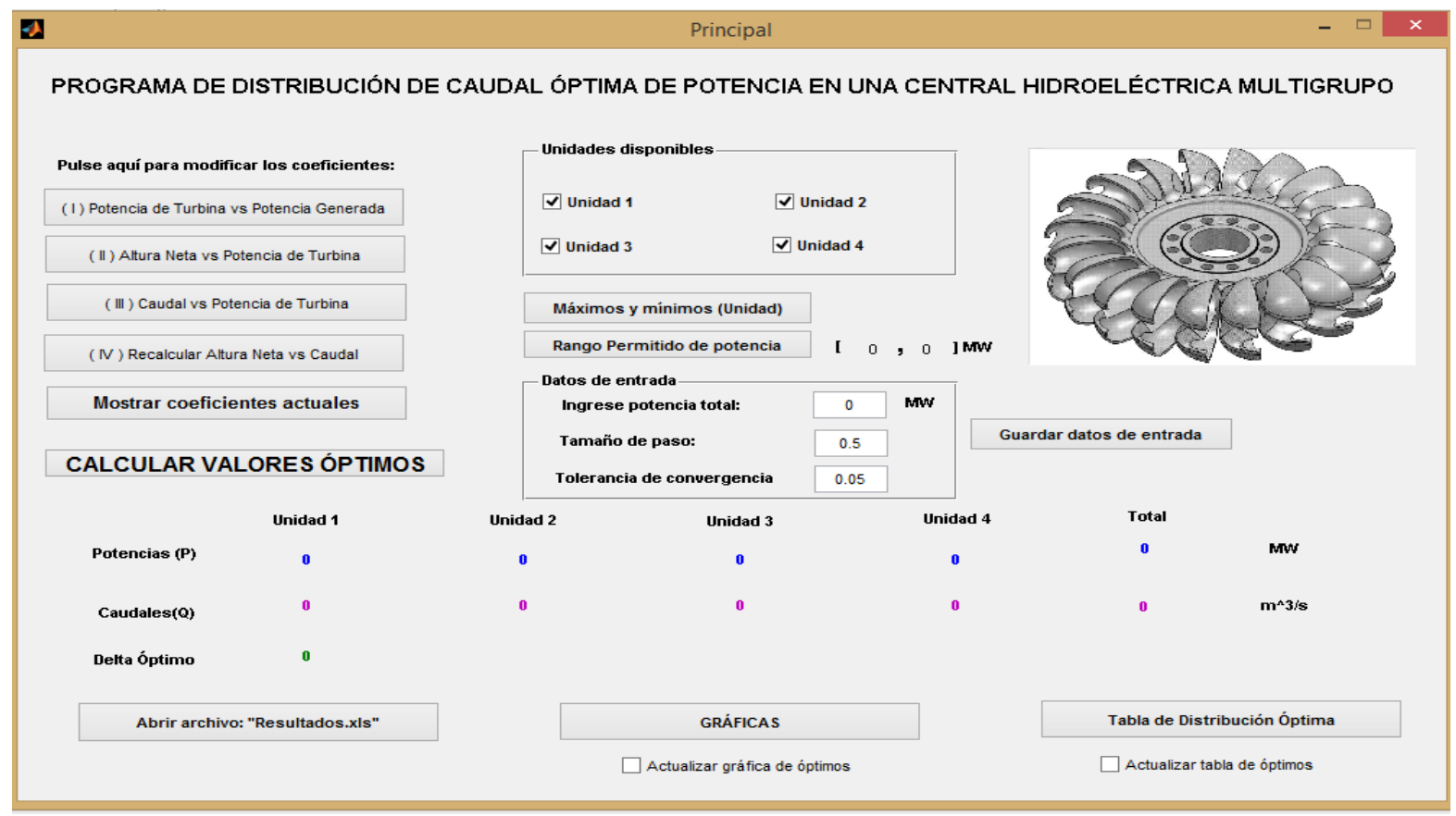

Fig. 6 Panel Principal.

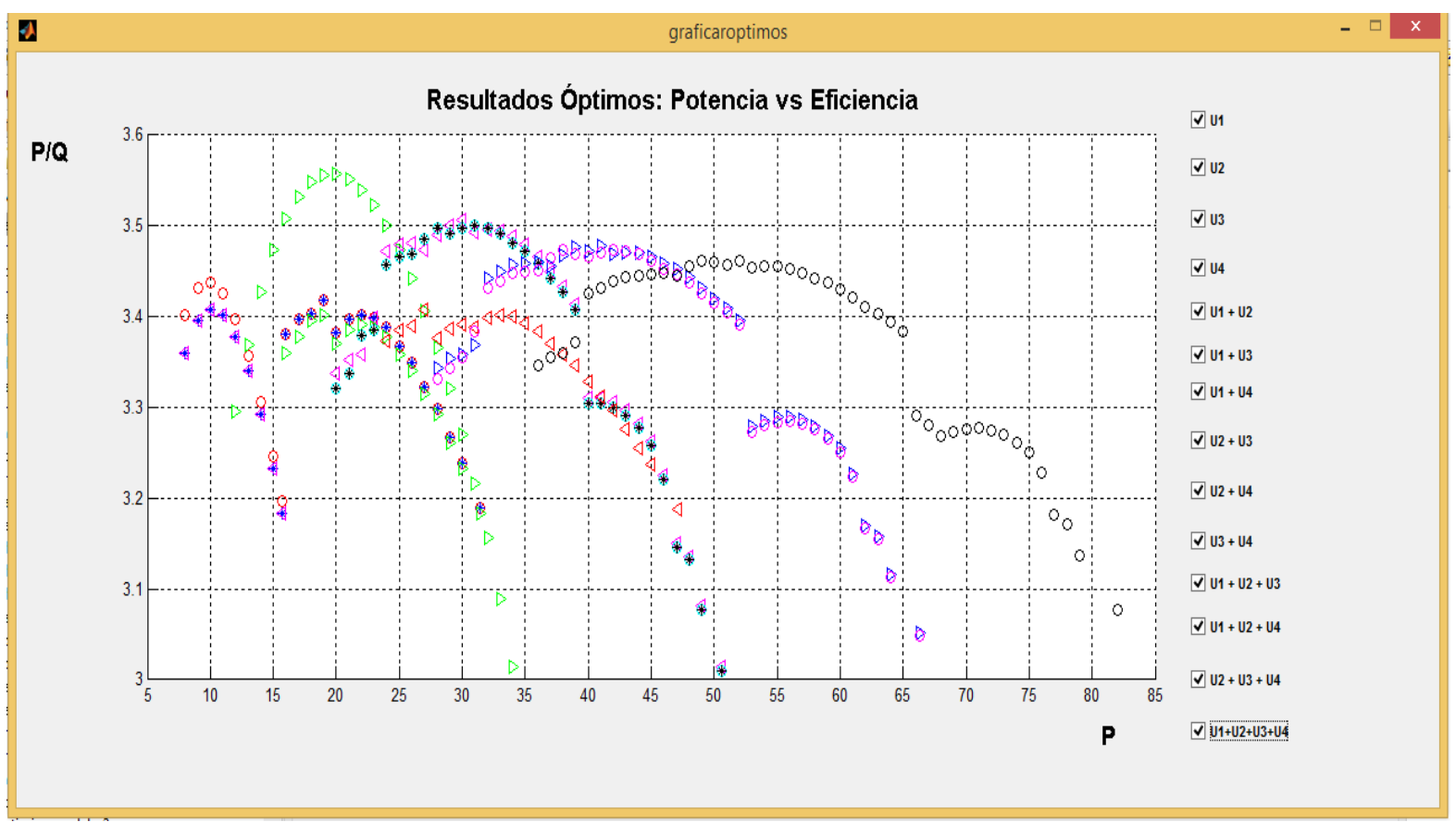

Fig. 7 Visualización gráfica del espectro de rendimientos $P / Q$ vs $P$ por grupos y combinaciones. 


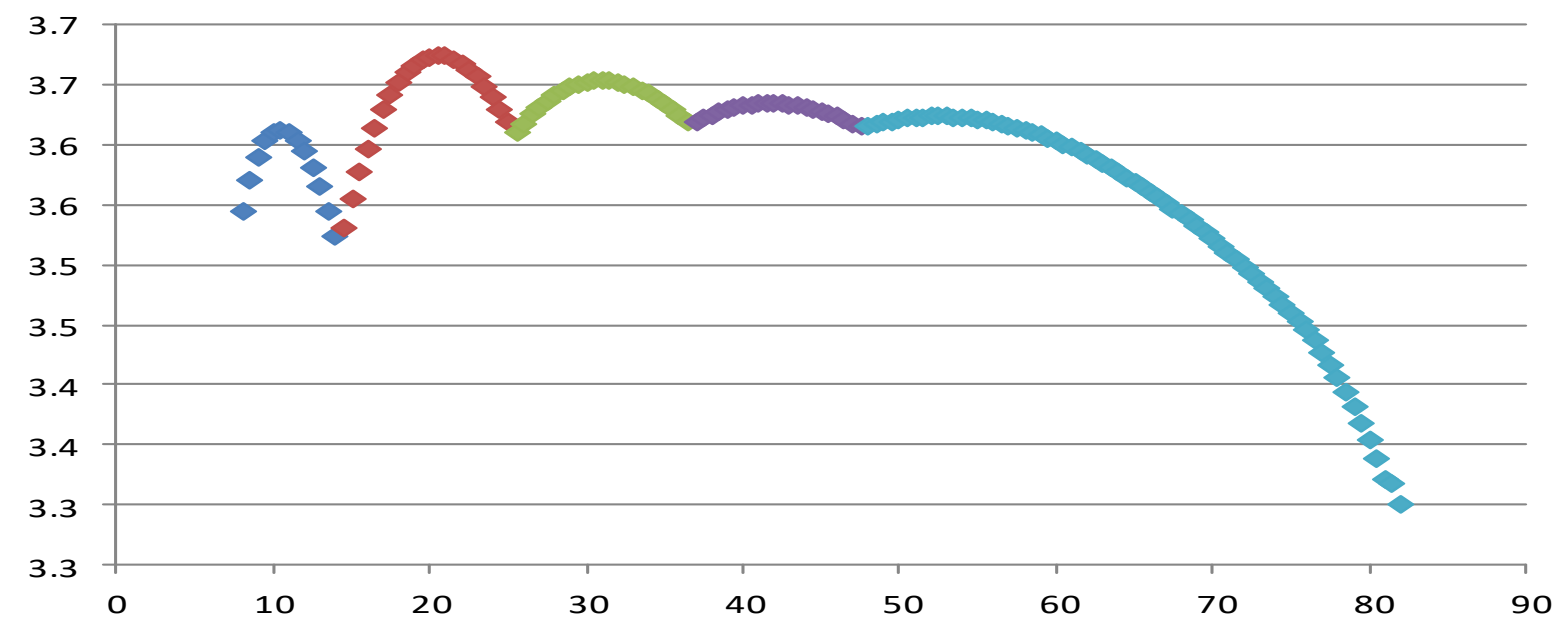

Fig. 8 Salida exportada a Excel de la curva óptima de rendimiento $P / Q$ vs potencia generada P de la C.H. Callahuanca.

\section{CONCLUSIONES Y RECOMENDACIONES}

- El método termodinámico de evaluación energética de la central hidroeléctrica, permite estimar los parámetros con una mayor aproximación que el método convencional, además incluye el efecto energético del cambio de temperatura entre la salida y entrada de cada turbina hidráulica.

- Se ha logrado desarrollar una metodología y aplicación informatizada de optimización de la potencia de una central hidroeléctrica multigrupo, tal que maximiza su eficiencia para cualquier condición de carga requerida a la central dentro de un programa diario de despacho, considerando incluso aquel escenario en que alguna unidad se encontrara fuera de servicio. Ello se demuestra con la aplicación específica a la Central Hidroeléctrica de Callahuanca.

- El proceso de optimización propuesto puede ser fácilmente adaptado a cualquier central hidroeléctrica que posea dos o más unidades de generación: utilizando el programa en Matlab desarrollado, solamente se tendría que introducir los parámetros energéticos de cada unidad de generación.

- En una central hidroeléctrica que poses grupos de generación gemelos, la eficiencia de placa de éstos llega a desbalancearse con el tiempo operando a cargas parciales, de forma tal que no van a generar potencias iguales, caudales iguales a rendimientos iguales. Entre las causas están el desfase de mantenimientos entre ambas unidades gemelas y el desbalance en las pérdidas transversales. Por lo tanto, es necesario aplicar la metodología propuesta con la finalidad de lograr el mejor ajuste de caudal y potencia operativa de cada unidad para la condición de máxima eficiencia.

\section{REFERENCIAS}

1. Díaz Cerna F. J. "Optimización de la operación y evaluación de la eficiencia técnica de una empresa de generación hidroeléctrica en mercados de corto plazo", Tesis Doctoral, Universidad Nacional de Colombia, 2011.

2. Baíllo A. "Optimización de la explotación y de la preparación de ofertas de una empresa de generación de energía eléctrica para mercados de corto plazo", Tesis Doctoral, Universidad Pontificia de Comillas, España, 2002.

3. Baíllo A., Ventosa M., Rivier M., Ramos A. "Optimal offering strategies for generation companies operating in electricity spot markets". IEEE Trans. Power Syst., vol. 19, no. 2, pp. 745753, May 2004.

4. Alarcón Jarpa I. A. "Método termodinámico para calcular el rendimiento en turbinas hidráulicas”, Universidad de Concepción, Chile, 2009.

5. Villasanti C. "Optimización del despacho de unidades generadoras en una central 
hidroeléctrica utilizando algoritmos evolutivos multiobjetivo", Universidad Católica Nuestra Señora de la Asunción, Paraguay, 2011.

6. Von Lucken Ch. "Optimización del despacho de unidades generadoras", Universidad Nacional de Asunción, Paraguay, 2008.

7. Arce A., Ohishi T., Soares S. "Optimal Dispatch of Generating Units of the Itaipú Hydroelectric Plant", IEEE Transactions on Power Systems, Volume 17, Number 1, February, 2002.

Correspondencia: miguelrosas@enel.com 